\title{
RF Rendez-Blue: Reducing Power and Inquiry Costs in Bluetooth- Enabled Mobile Systems
}

\author{
Eric S. Hall \\ enrique.hall@gmail.com \\ Charles D. Knutson \\ knutson@cs.byu.edu \\ David K. Vawdrey
}

Follow this and additional works at: https://scholarsarchive.byu.edu/facpub

Part of the Computer Sciences Commons

\section{Original Publication Citation}

Eric S. Hall, David K. Vawdrey, and Charles D. Knutson. "RF Rendez-Blue: Easing the Bluetooth Power and Delay Burdens in Mobile Systems." Proceedings of the Eleventh IEEE International Conference on Computer Communications and Networks (IC3N '2), Miami, Florida, October 14-16, 22.

\section{BYU ScholarsArchive Citation}

Hall, Eric S.; Knutson, Charles D.; and Vawdrey, David K., "RF Rendez-Blue: Reducing Power and Inquiry Costs in Bluetooth-Enabled Mobile Systems" (2002). Faculty Publications. 528.

https://scholarsarchive.byu.edu/facpub/528

This Peer-Reviewed Article is brought to you for free and open access by BYU ScholarsArchive. It has been accepted for inclusion in Faculty Publications by an authorized administrator of BYU ScholarsArchive. For more information, please contact ellen_amatangelo@byu.edu. 


\title{
RF Rendez-Blue: \\ Reducing Power and Inquiry Costs in Bluetooth-Enabled Mobile Systems
}

\author{
Eric S. Hall, David K. Vawdrey, and Charles D. Knutson \\ esh@byu.edu,dkv@email.byu.edu,knutson@cs.byu.edu \\ Computer Science Department \\ Brigham Young University \\ Provo, UT 84602
}

\begin{abstract}
In resource-limited mobile computing devices, Bluetooth wireless technology imposes a weighty burden due to ineffcient power utilization and a sluggish device discovery process. Buttressing Bluetooth with Radio Frequency Identifcation (RFID) technology by performing an operation we call "Rendez-Blue" alleviates these limitations. In the Rendez-Blue process, an RFID signal is used as a cue to "wake-up" a sleeping Bluetooth radio. This ensures that the Bluetooth radio is active only when needed, signifcantly reducing power consumption. In addition, RFID is used to communicate Bluetooth device information, allowing the user to bypass the traditional 10.24-second discovery process.
\end{abstract}

\section{INTRODUCTION}

$\mathbf{I}$ $\mathrm{N}$ tegration of Bluetooth wireless technology into mobile devices is increasingly prevalent. Bluetooth radios are rapidly making their way into mobile phones, notebook computers, and Personal Digital Assistants (PDAs). According to market projections, Bluetooth chipset shipments will increase dramatically, reaching sales of 780 million units in 2005 [1]. Amid the media hype and the rush to incorporate Bluetooth technology into a plethora of battery-powered products, some of Bluetooth's limitations have been ignored. Excessive power requirements and a sluggish discovery process impose a weighty burden upon resource-limited devices.

In Wireless Personal Area Networks (WPANs), Bluetooth is an excellent technology for providing connectivity between mobile systems that are limited in processing capabilities and power resources. Such devices require a low-overhead communication solution that does not restrict mobility, increase cost, or adversely affect form factor [2].

Minimizing power consumption is fundamental to extending mobility. When link establishment and data communication is not intended, keeping the Bluetooth radio "on" consumes power needlessly. This ineffcient use of power severely affects battery life and limits the usability of the device.

Another burden associated with Bluetooth is a lengthy discovery time. In some cases 10.24 seconds is simply too long to allow timely and ef£cient communication between mobile devices. There are certain usage models where the Bluetooth discovery delay places an unacceptable constraint upon a mobile user [3].

The problem of inef£cient power consumption and lengthy discovery time can be alleviated with the aid of low-power, low-overhead Radio Frequency Identifcation (RFID) technology [4]. Combining RFID with Bluetooth enables a hybrid transceiver that works as follows: An RFID module in a handheld device receives a signal from a nearby RFID host device. This signal is a "wake-up" cue to the sleeping Bluetooth radio on the handheld device. In response, the handheld device's RFID module communicates the necessary Bluetooth connection parameters, allowing the devices to establish a Bluetooth link without performing the normal discovery process. When communication is halted, the Bluetooth module on the handheld device returns to an inactive mode where it consumes virtually no power.

This paper discusses the benefts of combining Bluetooth with RFID to reduce the power and inquiry ineffciencies of Bluetooth technology in mobile systems. Section two discusses research that provides a foundation for the development of a hybrid device combining Bluetooth and RFID. Section three explains the power consumption diffculties and inquiry time limitations associated with integrating Bluetooth into mobile systems. Section four provides an overview of RFID technology. Section £ve describes Rendez-Blue, a process that uses RFID and Bluetooth in a single device to reduce power consumption and accelerate Bluetooth device discovery.

\section{FOUNDATIONAL WORK}

Prior research in wireless data communications has established the viability of using multiple transports in a device to alleviate limitations associated with a single technology. By combining IrDA with Bluetooth to aid in device discovery, researchers have illustrated a fourfold reduction in discovery time delays [5]. This research demonstrates some of the benefts of merging wireless technologies in a single device to leverage the strengths of each component.

Other research has shown that "node rendezvous" can be performed between embedded system "nodes" to conserve power [6]. Node rendezvous is accomplished using a low-overhead technology such as RFID to intelligently wake a more costly, higher performance communication device such as a Bluetooth radio. When communication is not desired, the higher power communication device can be turned off to reduce power consumption. To further extend battery life, other researchers have developed energy-conscious protocols designed to minimize the amount of time RF transceivers are active during a communication session [7].

Combining Bluetooth with an RFID transceiver addresses some of the challenges encountered during development of the 
Poket Doktor System at the Mobile Computing Laboratory of Brigham Young University [8]. The Poket Doktor incorporates a Bluetooth transceiver into a traditional smart card that is used to store a patient's medical information. In an emergency, medical workers can use handheld computers to wirelessly obtain data critical to patient care. The Poket Doktor usage model requires that the Bluetooth radio on the smart card be active only in an emergency situation. At other times the radio should remain inactive to save power and preserve battery life.

\section{Limitations OF BLUETOOTH}

\section{A. Inef£cient Power Management}

Managing power consumption in mobile devices is crucial because battery life is directly related to mobility [9]. Large, heavy batteries can extend operational life, but at the same time may limit portability [10]. There are two motivations driving the demand for ef£cient power-management schemes in mobile platforms: (1) Battery technology is not keeping pace with rapid advances (and corresponding power demands) in embedded processors and wireless communication technologies [11]; and (2) It is not realistic in some usage models to swap or recharge batteries as frequently as is required to power an "always-on" wireless radio module.

During active Bluetooth communication sessions, relatively high power consumption is to be expected. Between sessions, when communication is not intended, power consumed by the Bluetooth radio can be costly. Even when the radio is not active, by default it remains powered in order to respond to connection and inquiry requests from other Bluetooth devices. The modes of operation for a Bluetooth device are shown in Figure 1.

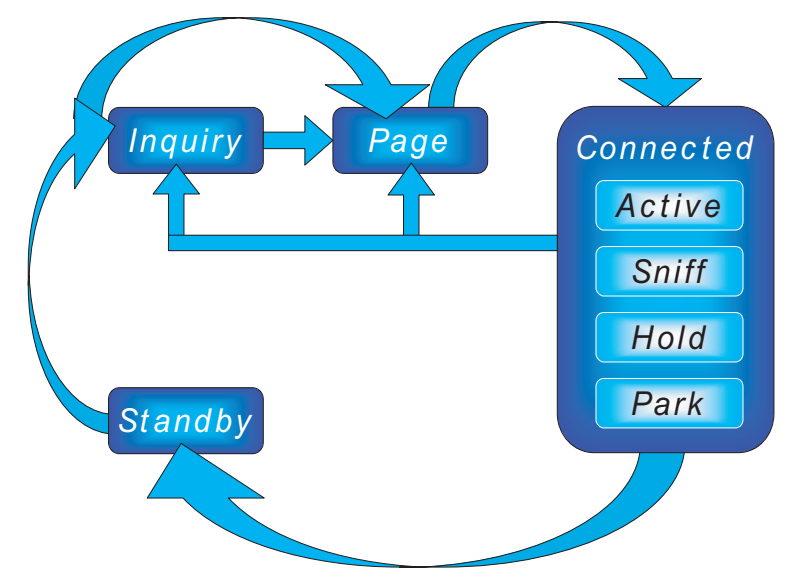

Figure 1. Standard Bluetooth modes of operation.

In Bluetooth's default idle state, only the device's native clock runs, and all other components remain inactive. During the connection process, a device passes through inquiry and page modes while establishing a link. However, the inquiry procedure may be bypassed if the identity of the device to be paged is known. Once a device is in the connected state, it uses one of the following power modes: active, sniff, hold, or park.

In the connected state, each Bluetooth device assumes the role of either master or slave. A piconet is a connection between one master and up to seven active slaves. The master determines the frequency hopping pattern of the piconet and allocates bandwidth to each slave device. The device acting as master consumes more power than a slave because it is more active in transmitting within the piconet. After entering a connected state, a device may return to either inquiry or page mode to allow additional devices to join the piconet, or the device may return to idle mode when the piconet is disbanded [12].

The demand for power is highest when a Bluetooth device is in the connected state. Power consumed while connected is justifable because desired, useful data communication is taking place. Although sniff, hold, and park are designated as power management states, the average current draw in these modes is still signifcant for use in mobile devices. Compared with standby and shutdown, even idle mode consumes a large amount of power although no active communication is taking place. This is undesirable if other Bluetooth devices are not accessible, or communication with nearby devices is not desired.

In addition to Bluetooth's idle mode, various low power "standby" modes are also available. The Ericsson 101008 Bluetooth module (see Figure 2) reveals a large variance in the power consumed for each of these states [13]. In idle mode, the average current draw is $15 \mathrm{~mA}$ following a Host Controller Interface (HCI) reset or $23 \mathrm{~mA}$ following a hardware reset. Two software standby modes offer considerable power savings but an external signal is required to awaken the module. The frst allows for wake-up via UART, and the second allows wake-up using either UART or RF. Completely shutdown, the Bluetooth hardware requires merely $1 \mu \mathrm{A}$ average current.

Power savings achieved using software standby modes is signifcant when compared to remaining in idle mode between communications. The complication associated with the software standby modes is the requirement that a wake-up signal come from an external source such as a microcontroller. In the case of wake-up via UART, either 1) the local device must wake up the radio at timed intervals and allow polling for Bluetooth devices in range, or 2) the local device must have knowledge that a Bluetooth device is within range and desires communication before issuing the wake-up signal.

The second option provides for the best power management scheme. Although a 1.25 second start-up delay is required to bring a device out of shutdown, the hardware shutdown state is the most power-ef£cient mode. Furthermore, the $1 \mu \mathrm{A}$ required in hardware shutdown mode allows Bluetooth to have virtually no impact upon the battery life of a mobile host during periods when communication is not taking place or intended. Similar to waking up the radio from software standby, the optimal method for bringing the radio out of shutdown mode involves knowledge that a Bluetooth device is within range and desires communication. Maximizing the time spent in hardware shutdown, and software standby modes signifcantly reduces the power demands associated with Bluetooth integration into mobile systems.

\section{B. Duration of Inquiry}

Another challenge associated with Bluetooth is its lengthy device discovery time. In some cases 10.24 seconds [12] is too long to allow timely communication between mobile devices. Envision a scenario where two devices approach each 


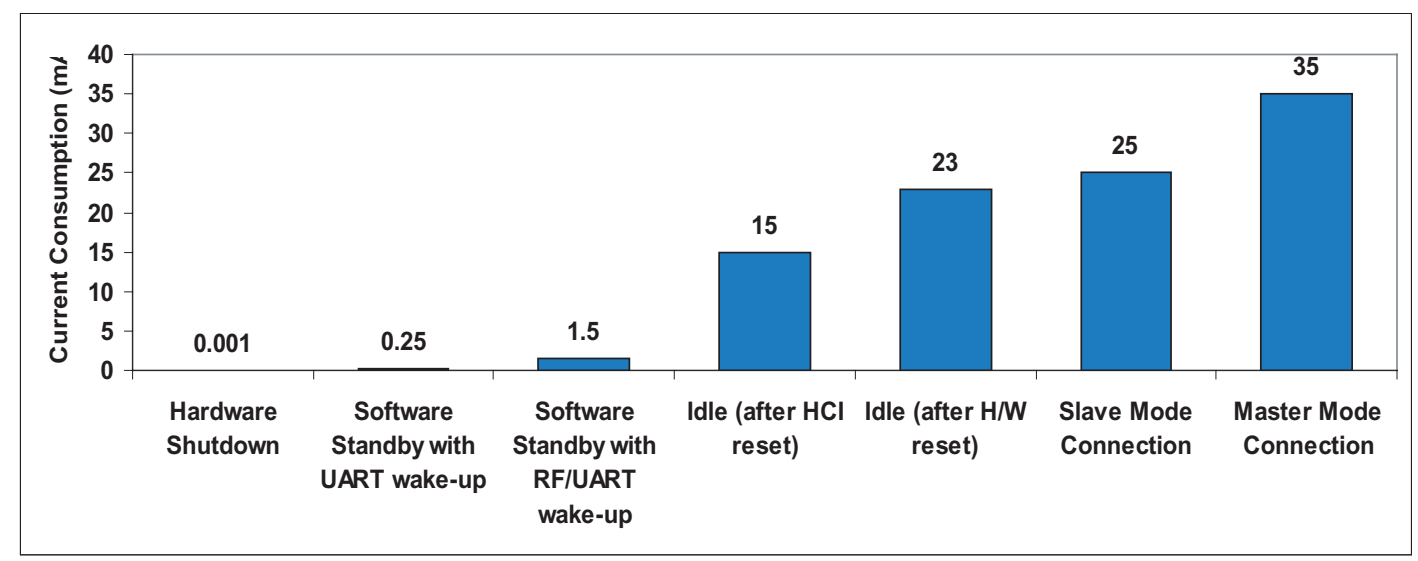

Figure 2. Power consumption in various Bluetooth modes.

other from opposite directions and wish to communicate. Using the standard Bluetooth mechanism, the devices may cross paths and be out of range before the device discovery process is complete. A lengthy discovery process is also unacceptable in time-intensive usage models such as Point-of-Sale (POS) transactions[14]. Delays in device discovery and link establishment may frustrate consumers and discourage the use of Bluetooth at POS terminals. The current implementation of Bluetooth device discovery is not satisfactory in these situations. The reasons for the sometimes unacceptable delay are discussed in the following sections.

1) Inquiry and Scanning: Bluetooth discovery requires two devices, one in inquiry mode and the other in inquiry scan mode. The device in inquiry mode actively seeks other devices by transmitting ID packets which contain an Inquiry Access Code (IAC). The inquiring device transmits every 312.5 $\mu \mathrm{s}$, twice the normal slot rate, in an effort to locate scanning devices. The inquiry scanning device listens for these ID packets while hopping every 2,048 slots, or every 1.28 seconds.

Upon receiving an ID packet, the scanning device does not immediately respond; rather, a random back-off is employed lasting up to $640 \mathrm{~ms}$. This delayed response prevents the inquiring device from being overwhelmed with replies from multiple inquiry scanning devices. The random back-off also helps to prevent a surge of radio traffc in the $2.4 \mathrm{GHz}$ ISM (Industrial, Scientifc, and Medical) band from multiple simultaneous responses. After backing off, the scanning device listens for a second ID packet.

When a second inquiry signal is received, the scanning device responds with a Frequency Hop Synchronization (FHS) packet providing relevant information for establishing a connection. Most importantly, the FHS packet contains the host Bluetooth device's hardware address and clock information. The FHS response packet covers a single time slot. The inquiring device always listens at the last frequency that it used to send an ID packet, so that it will be ready to receive an FHS response from a scanning device.

Some scenarios complicate the Bluetooth device discovery process. Since the inquiring device hops at twice the normal communication rate, receiving an FHS response in the frst half of a time slot prevents another slave from responding in the second half of the time slot because the inquiring device is unable hop to the second frequency quickly enough. Likewise, receiving an FHS response in the second half of a slot prevents the inquiring device from receiving a response in the frst half of the next transmission slot.

In an error-free environment, the inquiry process takes 10.24 seconds to insure coincidence of all devices in range. The discovery process uses 32 of Bluetooth's 79 channels. These 32 channels are divided into two 16-channel "trains," each of which require $10 \mathrm{~ms}$ to traverse. Guaranteeing coincidence based on statistical models requires each train to be traversed 256 times, and this train traversal process to be repeated at least twice for each train, requiring a total of 10.24 seconds:

$$
2 \text { trains } \cdot 256 \text { travs } \cdot 2 \text { iterations } \cdot 0.01 s=10.24 \mathrm{~s}
$$

This process guarantees discovery in an error-free environment; however, discovery amidst interference may require additional iterations of the train traversal process to discover all Bluetoothenabled devices within range [12].

2) Paging: During the paging process, the roles of master and slave are determined and the frequency hopping pattern of the devices is synchronized. Like the inquiry process, paging involves one device (in page scan mode) listening for ID packets, and the other (in page mode) issuing ID packets and waiting for a response. The paging device sends packets with the target device's address (which it obtained from an FHS packet received during inquiry). There is no need for a back-off mechanism during paging, since the ID packet sent by the pager is sent to a specifcally addressed device.

When the listening device receives this ID packet, it replies with its own ID packet. This response is acknowledged by the pager with an FHS packet, which elicits an ID packet reply from the listener. The FHS packet provides clock information that allows the page scanner to calculate the pager's hop sequence. After this exchange, both devices enter the connected state with the pager assuming the role as master and the listening device becoming the slave. 
The inquiry process may be bypassed if the hardware address of the device to be discovered is already known. With this information, the process of obtaining the FHS packet from the inquiry scanning device is unnecessary and the device can move from idle mode directly into page mode. Once paging begins, the devices can establish their respective roles and synchronize hopping in the normal fashion. Thus, bypassing inquiry mode removes the potential 10.24 second delay in connection establishment.

\section{RFID OVERVIEW}

RFID is a low-power, low-bandwidth wireless communication technology. An RFID system is composed of an antenna or coil, a transceiver, and a transponder (known as an RF tag). Signals are directed from a transceiver to a mobile tag, allowing close-proximity electromagnetic or inductive coupling to transmit a small amount of data (see Figure 3). Range is primarily determined by the power output and frequency used, and can be extended to about 30 meters [4].

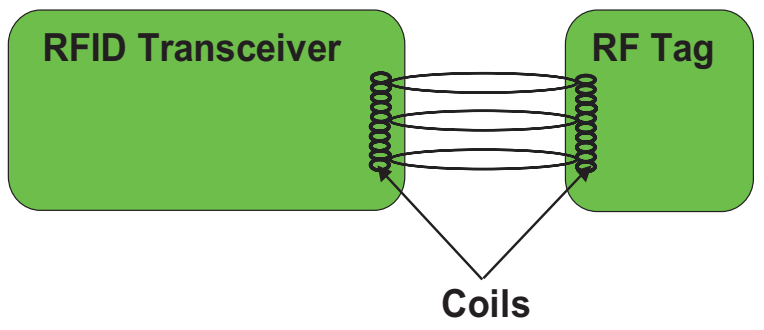

Figure 3. Inductive coupling in RFID communication.

RF tags come in two classifcations: active and passive. Active tags allow for modest amounts of data storage (up to $1 \mathrm{MB}$ ) and have read/write capabilities. Active tags also require an internal power source and consequently place a battery burden upon a mobile host. Passive tags do not require an additional power supply since they obtain operating power from the transmitted signal. Thus, passive tags offer a virtually unlimited operational lifetime, even in a mobile host with limited resources. Passive tags are small, lightweight, and inexpensive. They are typically read-only devices, storing only a limited amount of data (32 to 128 bits). Furthermore, the transceiver communicating with a passive RF tag often requires more power since it must supply the passive tag with operating power.

\section{RF RENDEZ-BLUE}

The technique of placing a high-power radio in a powereddown state, and waking it only to communicate has been referred to as a "node rendezvous" [6]. Using this technique prevents the constant power drain by a communications module when it is not in use. Node rendezvous can be achieved by externally triggering a low-power device to awaken a higherpower Bluetooth radio. Research suggests that RFID is an ideal technology for facilitating this process [15]. Additionally, RFID can also be used to communicate Bluetooth connection parameters, such as the device hardware address, in order to bypass the sluggish inquiry process. This section introduces an RFID/Bluetooth hybrid device which demonstrates signifcant power savings and accelerated device discovery. The process referred to as Rendez-Blue involves the transfer of Bluetooth device information to accelerate discovery using node rendezvous.

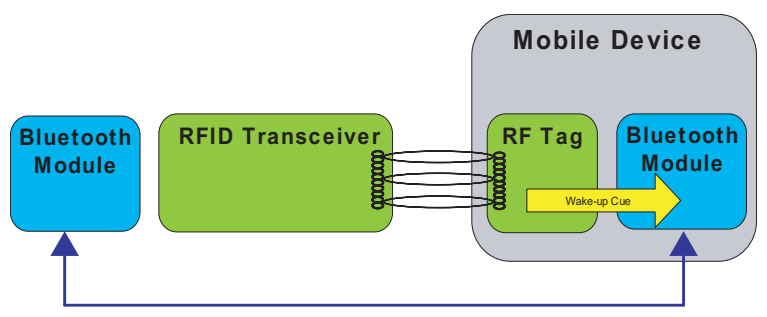

Figure 4. Node rendezvous using RFID to "wake-up" the Bluetooth radio.

\section{A. Solving the Power Problem}

RFID provides intelligent external signals to bring the Bluetooth radio out of software standby or hardware shutdown modes (see £gure 4). Using RFID allows the Bluetooth device to minimize time spent in idle mode, which extends battery life in a mobile host.

Table I compares several batteries that are available for powering mobile devices [16]. The size, shape, and capacity of these batteries redect the intentions of their design. The Rendez-Blue process demonstrates how the operational life of such batteries can be signifcantly extended in certain usage models.

Large batteries, such as nickel metal hydride models, are used to power cellular phones and PDAs and can satisfy substantial power demands for moderate lengths of time. For example, a typical cellular phone battery can support active Bluetooth communication for 42 continuous hours. The tradeoff for greater power capacity is increased size and weight, which limits the number of suitable uses for such a battery.

In Bluetooth-enabled PDAs and cellular phones, the Bluetooth radio typically acts as a peripheral communications module, not as the primary power consumer. Nevertheless, in these devices, Rendez-Blue removes the power demands of the Bluetooth radio when no communication is desired. This power savings is signifcant since supporting a Bluetooth module in idle mode would deplete a cell phone battery in roughly three days. Implementing Rendez-Blue allows the device to operate normally, conserving power but still offering Bluetooth functionality with minimal intervention. Limited resources are not siphoned to support an idle Bluetooth radio.

Smaller batteries, such as oxygen sensor cell and lithium button cell, are much more limited in storage capacity. Rechargeable lithium button cell batteries, for example, are ideal for devices such as cameras and toys that can be used for a duration and then recharged. Incorporating Rendez-Blue in Bluetooth devices using these batteries provides a substantial increase in battery life. A Bluetooth device in idle mode would drain the lithium battery in only two hours. In hardware shutdown mode, however, the battery can support an inactive radio for 30,000 hours-nearly three and a half years. If the Bluetooth radio is active only when needed, a lithium button cell battery can support 
TABLE I

EFFECT OF BLUETOOTH OPERATIONAL MODES ON BATTERY LIFETIME (ACTIVE MODE ASSUMES THE DEVICE IS OPERATING AS SLAVE).

\begin{tabular}{||c|c|c|c|c||}
\hline Battery & $\begin{array}{c}\text { Lithium } \\
\text { Flat Cell }\end{array}$ & $\begin{array}{c}\text { Rechargeable Lithium } \\
\text { Button Cell }\end{array}$ & $\begin{array}{c}\text { Oxygen } \\
\text { Sensor Cell }\end{array}$ & $\begin{array}{c}\text { Nickel Metal Hydride } \\
\text { Cellular Phone Battery }\end{array}$ \\
\hline \hline Dimensions (mm) & $0.4 \times 22 \times 29$ & $20 \times 2.5$ & $11.6 \times 5.4$ & $113 \times 44 \times 20$ \\
\hline Weight(g) & 0.5 & 2.5 & 1.85 & 150 \\
\hline Capacity (mAh) & 25 & 30 & 400 & 1,050 \\
\hline Slave Mode Lifetime (h) & 1 & 1.2 & 16 & 42 \\
\hline Idle Mode Lifetime (h) & 1.67 & 2 & 26.67 & 70 \\
\hline $\begin{array}{c}\text { Software Standby from } \\
\text { RF Wake-Up Lifetime (h) }\end{array}$ & 16.67 & 20 & 266.67 & 700 \\
\hline $\begin{array}{c}\text { Software Standby from } \\
\text { UART Wake-Up Lifetime (h) }\end{array}$ & 100 & 120 & 1,600 & 4,200 \\
\hline Hardware Shutdown Lifetime (h) & 25,000 & 30,000 & 400,000 & $1,050,000$ \\
\hline \multicolumn{2}{|c|}{}
\end{tabular}

active communication for several minutes each day over several weeks before being recharged. In this case, sporadic Bluetooth communication can take place over an extended period of time. Without Rendez-Blue, battery resources are depleted after only two hours, whether or not active communication is desired.

Examination of small lithium aat cell batteries demonstrates the benefts of Rendez-Blue in infrequently used devices. A Bluetooth-enabled medical card (such as the Poket Doktor Smart Card [8]) that communicates only in emergencies must be reliable without battery charging or replacement. The fully charged dat cell contains enough power to support active communication for about one hour. Without Rendez-Blue, Bluetooth operating in idle mode would drain battery resources in only about 1.5 hours. Incorporating Rendez-Blue extends the operational lifetime (for the inactive radio) to over 20,000 hours-more than two years. In a situation where the card is used occasionally to transmit medical information, Rendez-Blue enables the battery to power several minutes of active communication distributed in various sessions over a period of a year and a half.

Research demonstrates that the Rendez-Blue process is able to conserve power in a number of applications using a variety of power sources. Rendez-Blue stretches the active communication time of a resource-limited device over an extended duration, interweaving short active sessions with longer periods in standby or shutdown mode.

\section{B. Dealing with Discovery}

Passive RFID tags can transmit up to 128 bits of data, suf£cient to communicate the Bluetooth device discovery information required to bypass the inquiry phase. The fully-encoded FHS payload has a gross length of 240 bits; however, most of this information (including clock data, parity codes, and CRC data) does not need to be transmitted in order to bypass the inquiry process. The critical information to transfer is the mobile device's unique 48 bit MAC address, also known as the Bluetooth Device Address (BD_ADDR). Since this address is static, it can be hard-coded into the RFID tag.

Once the passive tag hybrid receives an interrogation from the transceiver, it replies immediately via RFID with its local BD_ADDR (See Figure 5). This exchange replaces the lengthy
Bluetooth inquiry process by directly providing address information that would otherwise have been transmitted in an FHS packet. Following the exchange, the system can bypass inquiry and immediately initiate the paging process. The mobile device with the passive RFID tag supplies the BD_ADDR, and assumes the role of slave during the connection.

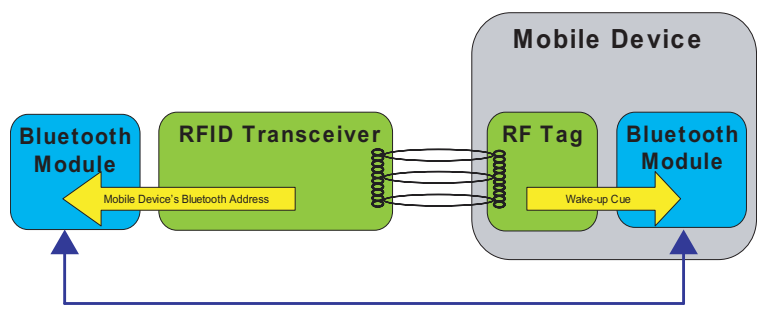

Figure 5. RFID communicates FHS data required to bypass inquiry process.

The comparison of connection times displayed in Figure 6 shows the time saved by using Rendez-Blue. The time required for exchange of the BD_ADDR between RFID modules is nominal. Related research indicates that the average time to complete Bluetooth paging is 1.8 seconds [5]. Thus, establishing a typical Bluetooth connection (including inquiry and paging) requires 12.0 seconds. Using RFID to bypass inquiry and enter directly into paging mode requires only 1.8 seconds, $85 \%$ less than the typical Bluetooth connection.

Adding the 1.25 seconds required to awaken the Bluetooth radio from the most power-ef£cient hardware shutdown mode [13], we determine that a device can enter a connected state from hardware shutdown using Rendez-Blue in 3.1 seconds. This represents a $75 \%$ time savings compared to a standard connection. The slight increase in connection time $(1.8 \mathrm{sec}-$ onds to 3.1 seconds) is a tradeoff associated with using the most power effcient Bluetooth shutdown mode rather than idle mode. Rendez-Blue results in a fourfold to sixfold reduction in the time required for mobile systems to establish a Bluetooth connection.

\section{Implications of Using an RFID/Bluetooth Hybrid}

Although the Rendez-Blue process signifcantly reduces the power and connection time constraints associated with Blue- 


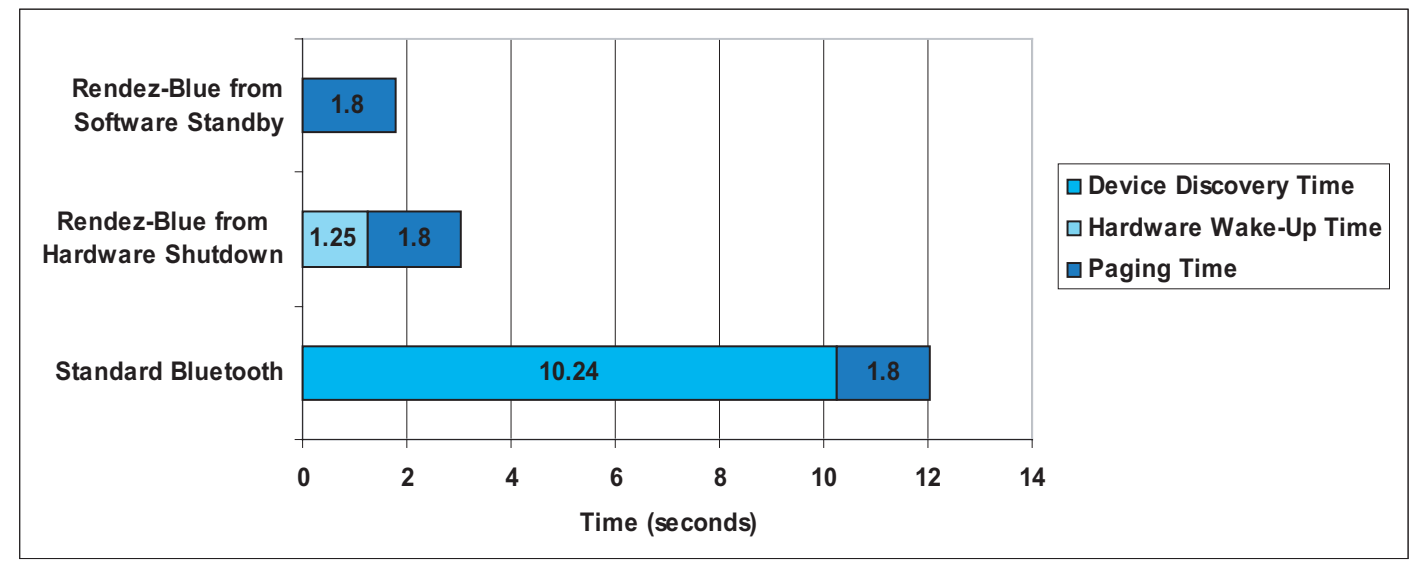

Figure 6. Comparison of time required to establish a Bluetooth connection.

tooth radios in mobile devices, some limitations are associated with the hybrid module. In a point-to-point Bluetooth communication scenario, both devices must be equipped with Bluetooth and RFID modules. One device must use a passive RF tag, and the other an active RFID transceiver. Connection establishment is dependent on this system architecture. Typically the most power-sensitive device should be the one with the passive RFID tag. Optimally, the device bearing the RFID transceiver is either stationary and not resource limited, or it is less dependent upon power resources than the device with the passive tag.

Another limitation of Rendez-Blue is the forced assignment of the slave role to the mobile device. While slave mode requires less power, it also limits the device to one active connection at a given time, whereas in master mode, the device could support up to seven active connections. It is possible to perform a role reversal between two devices (where the master assumes the slave role), but this increases the power demands of the resource-limited device. These tradeoffs must be evaluated by system designers to determine if the benefts of using Rendez-Blue outweigh the restrictions imposed by the process.

\section{CONCLUSIONS}

This paper has addressed methods for increasing the usability of Bluetooth wireless technology in resource-constrained mobile devices. Using the Rendez-Blue process to wake up sleeping hardware, the impact of Bluetooth technology on battery life is minimized. As a result, Bluetooth-enabled devices may function using smaller batteries, and may distribute their active communication sessions over longer periods of inactivity. Rendez-Blue can also be used to accelerate the traditional Bluetooth inquiry process. Using RFID to transmit Bluetooth hardware address information results in a four-to-sixfold reduction in device discovery time.

\section{REFERENCES}

[1] J. Putscher, "Bluetooth chips kick butt," Cahners In-Stat Group, Scottsdale, Arizona, December 5, 2001.

[2] I. Chlamtac, J. Redi, "Mobile computing: Challenges and potential," Encyclopedia of Computer Science, 4th Edition, International Thomson Publishing, 1998.

[3] T. Salonidis, P. Bhagwat, L. Tassiulas, "Proximity awareness and fast connection establishment in Bluetooth," Proceedings of the First Annual ACM Worshop on Mobile and Ad Hoc Networking and Computing, Boston, Massachusetts, August 11, 2000.

[4] Association of the Automatic Identifcation and Data Capture Industry, "Radio Frequency Identifcation: RFID a basic primer," White Paper, 2001.

[5] R. Woodings, D. Joos, T. Clifton, C. D. Knutson, "Rapid heterogeneous connection establishment: Accelerating Bluetooth inquiry using IrDA," Proceedings of the Wireless Communications and Networking Conference (WCNC), Orlando, Florida, March 19, 2002.

[6] T. Todd, F. Bennett, A. Jones, "Low power rendezvous in embedded wireless networks." Proceedings of the First IEEE Annual Workshop on Mobile Ad Hoc Networking \& Computing (MobiHOC), Boston, Massachussetts, August 11, 2000.

[7] I. Chlamtac, C. Petrioli, J. Redi, "Energy conserving access protocols for identifcation networks," IEEE/ACM Transactions on Networking 7(1): 51-59, 1999.

[8] E. S. Hall, D. K. Vawdrey, C. D. Knutson, "The Poket Doktor: A Bluetooth healthcare application," Submitted to IEEE Engineering in Medicine and Biology Magazine, March 2002.

[9] D. Panigrahi, C. Chiasserini, S. Dey, R. Rao, A. Raghunathan, K. Lahiri, "Battery life estimation of mobile embedded systems," Proceedings of the 14th International Conference on VLSI Design, January 2001.

[10] R. A. Powers, "Batteries for low power electronics," Proceedings of the IEEE, 83: 687-93, April 1995.

[11] F. Koushanfar, V. Prabhu, M. Potkonjak, J. M. Rabaey, "Processors for mobile applications," Proceedings of the IEEE International Conference on Computer Design Abstract (ICCD), 603-8, October 2000.

[12] Bluetooth Special Interest Group, "Specifcation of the Bluetooth System v1.1," February 2001.

[13] Ericsson Microelectronics, "ROK 101008 Bluetooth module datasheet," September 2001.

[14] N. A. Thomas, "Infrared and Bluetooth transactions at the Point of Sale," In2M Corporation White Paper, 2001.

[15] M. Nosovic, T. Todd, "Low power rendezvous and RFID wakeup for embedded wireless networks," Proceedings of the 15th IEEE Annual Computer Communications Workshop $(\mathrm{CCW})$, Captiva Island, Florida, October 15-18, 2000.

[16] Varta, Battery Specifcations Document, Available from http://www.varta.com, 2002. 